\title{
Emprego da citologia vaginal na detecção da fase do ciclo estral de cadelas e sua relação com a idade e escore de condição corporal, Belém, Pará
}

\author{
Use of vaginal cytology in the detection of the estral cycle phase of bitches and its relation with age \\ and body condition score, Belém, Pará \\ Uso de la citología vaginal en la detección de la fase del ciclo estral de las perras y su relación con la \\ edad y el estado corporal, Belém, Pará
}

\author{
Giovanna Pinheiro Oliveira \\ ORCID: https://orcid.org/0000-0002-7339-8000 \\ Universidade da Amazônia, Brasil \\ E-mail: giovannavet98@gmail.com \\ Heider Felipe Figueiredo de Souza \\ ORCID: https://orcid.org/0000-0002-6098-2435 \\ Universidade da Amazônia, Brasil \\ E-mail: souzaheider_11@hotmail.com \\ Daniel Pereira Batista \\ ORCID: https://orcid.org/0000-0002-0510-8200 \\ Universidade da Amazônia, Brasil \\ E-mail: danbatita5146@gmail.com \\ Aryane Silva \\ ORCID: https://orcid.org/0000-0002-3279-703X \\ Universidade da Amazônia, Brasil \\ E-mail: anemedvet@hotmail.com \\ Welligton Conceição da Silva \\ ORCID: https://orcid.org/0000-0001-9287-0465 \\ Universidade Federal Rural da Amazônia, Brasil \\ E-mail: welligton.medvet@gmail.com \\ Lílian Kátia Ximenes Silva \\ ORCID: https://orcid.org/0000-0002-0790-1958 \\ Universidade da Amazônia, Brasil \\ E-mail: silva_lilian@yahoo.com.br
}

\begin{abstract}
Resumo
A realização da citologia vaginal permite detectar a fase do ciclo estral e distúrbios reprodutivos em cadelas. O objetivo do estudo foi realizar o emprego da citologia vaginal na detecção da fase do ciclo estral de cadelas (Canis lupus familiaris) no município de Belém, no estado do Pará. Os exames citológicos vaginais foram previamente autorizados pelos tutores, sendo realizados em 23 cadelas, sem raça definida (SRD), com idade entre 6 meses a 14 anos, pesando de 5 a $25 \mathrm{~kg}$, antes de serem submetidas a ovariosalpingohisterectomia (OSH). Os resultados demonstraram que 13,04\% (3/23) das fêmeas estavam em proestro; 21,73\% (5/23) em estro; 39,13\% (9/23) em diestro e 26,08\% (6/23) em anestro. Dessa forma, demonstrou-se que a citologia vaginal pode ser empregada na rotina clínica de fêmeas caninas para diagnóstico da fase do ciclo estral, ressaltando-se que a associação com a avaliação clínica e hormonal favorece maior precisão nesse diagnóstico.
\end{abstract}

Palavras-chave: Diagnóstico; Exame citológico; Fêmeas caninas; Reprodução.

\begin{abstract}
Vaginal cytology allows the detection of the estrous cycle phase and reproductive disorders in bitches. The objective of the study was to perform the use of vaginal cytology to detect the estrous cycle phase in female dogs (Canis lupus familiaris) in the city of Belém, Pará State, Brazil. The vaginal cytological exams were previously authorized by the guardians, being carried out in 23 female dogs, without defined breed (SRD), with age between 6 months and 14 years, weighting from 5 to $25 \mathrm{~kg}$, before being submitted to ovariosalpingohysterectomy (OSH). The results showed that $13.04 \%(3 / 23)$ of the females were in proestrus; $21.73 \%$ (5/23) in estrus; $39.13 \%$ (9/23) in diestrus and 26.08\% $(6 / 23)$ in anestrus. Thus, it was demonstrated that vaginal cytology can be used in the clinical routine of canine females for diagnosis of the estrous cycle phase, emphasizing that the association with clinical and hormonal evaluation favors greater accuracy in this diagnosis.
\end{abstract}

Keywords: Diagnosis; Cytological examination; Canine females; Reproduction. 


\section{Resumen}

La citología vaginal permite detectar la fase del ciclo estral y los trastornos reproductivos en las perras. El objetivo del estudio fue realizar el uso de la citología vaginal para detectar la fase del ciclo estral en perras (Canis lupus familiaris) en la ciudad de Belém, Estado de Pará, Brasil. Los exámenes citológicos vaginales fueron previamente autorizados por los tutores, siendo realizados en 23 perras, sin raza definida (SRD), con edad entre 6 meses y 14 años, con peso de 5 a $25 \mathrm{~kg}$, antes de ser sometidas a ovariosalpingohisterectomía (OSH). Los resultados mostraron que el 13,04\% (3/23) de las hembras estaban en proestro; el 21,73\% (5/23) en estro; el 39,13\% (9/23) en diestro y el 26,08\% (6/23) en anestro. Así, se demostró que la citología vaginal puede ser utilizada en la rutina clínica de las hembras caninas para el diagnóstico de la fase del ciclo estral, destacando que la asociación con la evaluación clínica y hormonal favorece una mayor precisión en este diagnóstico.

Palabras clave: Diagnóstico; Examen citológico; Hembras caninas; Reproducción.

\section{Introdução}

A importância da detecção da fase do ciclo estral em cadelas é fundamental, pois se houver falhas pode induzir a erros que comprometem a cobertura das fêmeas, reduzindo a taxa de fertilização e consequentemente de nascimento (Oliveira, 2018). A citologia vaginal é uma técnica laboratorial prática e de baixo custo que auxilia na avaliação ginecológica de cadelas, detectando a fase do ciclo estral das mesmas, podendo ser introduzido na rotina clínica veterinária (Reynaud et al, 2005; Klein, 2014).

Nessa técnica são encontradas diferentes tipos celulares que correspondem as fases do ciclo estral da fêmea, como as células parabasais, intermediárias, superficiais e cornificadas (Allison et al., 2008; Solano-Gallego; Masserdotti, 2016). Portanto, a citologia vaginal identifica a fase do ciclo estral devido a alteração morfológica que as células epitelias vaginais sofrem durante o mesmo, de acordo com o nível de estrógeno circulante, passando de pequenas células arredondadas com citoplasma claramente visível em estágios não estrogênicos para células maiores, cornificadas e angulares com núcleo picnótico, sob a influência do estrógeno (Payan-Carreira et al., 2014; Vieira et al., 2012; Oliveira, 2018).

O ciclo estral das cadelas é compreendido pelo intervalo entre o período de desenvolvimento folicular seguido pela ovulação, ocorrendo em média a cada seis meses, ou seja, dois ciclos estrais por ano (Serafim, 2009; Silva, 2016). As fêmeas iniciam seu ciclo reprodutivo com o processo da puberdade, entre 5 a 12 meses de idade quando as fêmeas atingem $65 \%$ do peso corporal adulto, podendo ainda variar de acordo com a raça, tamanho e peso (Feldman; Nelson, 2004).

A classificação do ciclo estral da cadela é dividido em quatro fases: proestro, estro, diestro e anestro (KLEIN, 2014). O proestro tem duração média de 9 dias (Oliveira; Marques, 2006), e é caracterizado pelo desenvolvimento folicular com aumento da concentração de estrógeno, sintetizado pelas células da granulosa dos folículos, e que ocasiona a proliferação do epitélio vaginal (Solano-Gallego; Masserdotti, 2016).

O estro, é o período de receptividade sexual e é onde ocorre a ovulação, tem duração média de 10 dias (Jöchle; Andersen, 1997; Klein, 2014). A ovulação ocorre 24 a 48 horas após o pico de LH, nessa fase os valores de progesterona são elevados e de estrógeno mais baixos (Payan Carreira; Bessa, 2010). As características externas são a vulva edemaciada e corrimento vulvar menos abundante e mais claro, embora não seja uma regra para todas as cadelas, e as mesmas procuram os machos oferecendo a zona da cauda e exibindo a região perineal. Na citologia vaginal, observa-se predominância de células superficiais, que são poliédricas e com núcleo picnótico, além de intermediárias e bactérias (Buen De Argüero, 2001; Allison et al., 2008; Nogueira et al., 2019).

O diestro é também conhecido como a fase lútea, ou seja, de corpo lúteo ativo, com secreção intensa de progesterona, tem duração média de 65 dias e inicia-se quando a fêmea rejeita o macho (Musolino et al., 2000). As características externas nessa fase são discretas. Na citologia vaginal, há presença de células parabasais e intermediárias arredondadas com reduzida quantidade de bactérias e neutrófilos (Fontbonne; Malandain, 2006; Nogueira et al, 2019). 
O anestro é o período da inatividade sexual, que tem duração média de 120 dias (Alves et al., 2002; Chirinea, 2008), iniciando-se com a regressão do corpo lúteo e terminando com a próxima fase folicular (Feldman; Nelson, 2004). Nessa fase o corpo lúteo do ciclo anterior é visível e inativo e os níveis de estrógeno e progesterona encontram-se com valores basais. Há predomínio de células parabasais e intermediárias, e raramente neutrófilos e bactérias (Vieira; Oliveira, 2012).

Com base nessas informações, o objetivo neste estudo foi realizar o emprego da citologia vaginal na detecção da fase do ciclo estral de cadelas (Canis lupus familiaris) no municipio de Belém, no estado do Pará.

\section{Metodologia}

\section{1 Ética da pesquisa}

Previamente a realização da colheita do material citológico nas cadelas encaminhadas a clínica particular e ao Centro de Controle de Zoonoses (CCZ), todos os tutores foram devidamente consultados e e concordaram com a realização do procedimento.

\subsection{Local}

O estudo foi realizado em uma clínica particular e no Centro de Controle de Zoonoses (CCZ) no município de Belém $\left(1^{\circ} 27^{\prime} 21^{\prime \prime} \mathrm{S} 48^{\circ} 30^{\prime} 14^{\prime \prime}\right.$ O), no estado do Pará.

\subsection{Animais e histórico reprodutivo}

Foram utilizadas 23 ( $n=23$ ) fêmeas caninas, sem raça definida (SRD), com variação de idade entre 6 meses a 14 anos e peso de $5 \mathrm{a} 25 \mathrm{~kg}$. As fêmeas foram submetidas a anamnese e histórico reprodutivo (aplicação ou não de anticoncepcional), exame clínico, ginecológico e citológico, sendo avaliado também o escore de condição corporal (escala de 1 a 5).

\subsection{Colheita do material citológico}

A colheita de material para a citologia vaginal foi realizada antes das fêmeas serem submetidas a ovariosalpingohisterectomia (OSH).

Para a realização do exame citológico, a vagina das cadelas foi limpa com clorexidina (MARCA) externamente e quando necessário foi realizada a limpeza com algodão internamente em algumas fêmeas que apresentaram secreção sanguinolenta ou purulenta. O material foi obtido inserindo-se swab estéril em ângulo de $45^{\circ}$ e depois $180^{\circ}$, pressionando-se delicadamente na musculatura para a obtenção do material da região mais cranial da vagina (Figura 1A e B).

Figura 1. Swab estéril (A). Momento da coleta (B).
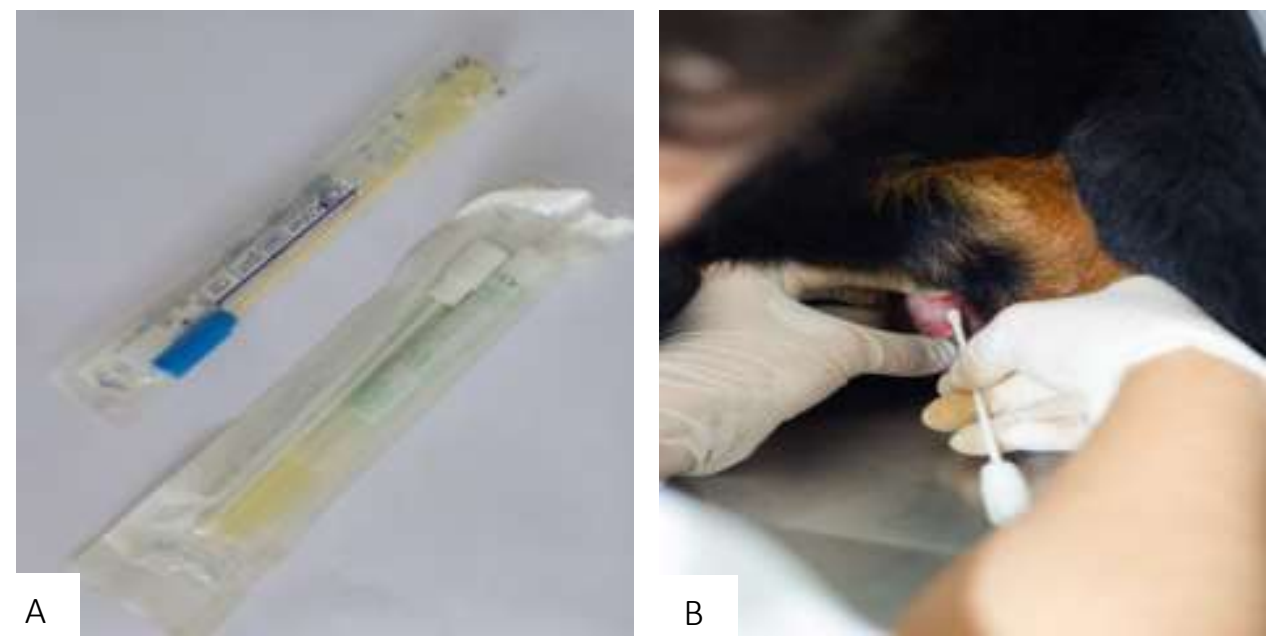

Fonte: Acervo dos autores. 
O material colhido foi fixado em lâminas identificadas (processo de imprint) e em seguida coradas com o Kit Panótico Rápido, sendo submersas nos 3 reagentes por um período de 60, 30 e 20 segundos, respectivamente (Figura 2). Posteriormente, as lâminas foram submetidas a secagem em temperatura ambiente, e analisadas em microscópio óptico (aumento de 40X) para identificação dos diferentes tipos celulares - parabasais, intermediárias e superficiais (anucleadas ou nucleadas).

Figura 2. Kit Panótico Rápido utilizado para coloração das lâminas.

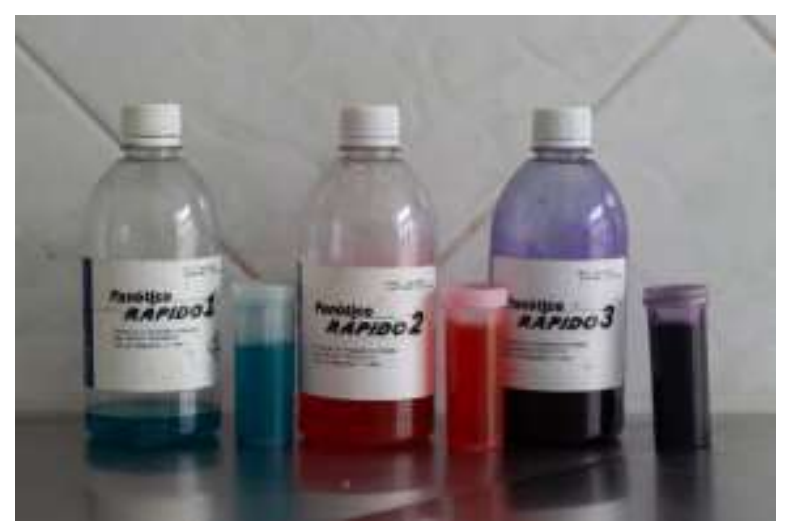

Fonte: Acervo dos autores.

\subsection{Análise estatistica}

Os dados foram organizados em planilhas do Microsoft Office ${ }^{\circledR}$ Excel 2014. Posteriormente, realizou-se a estatistica descritiva por meio do programa $\mathrm{R}$ versão 16.8 , sendo expostos os dados percentuais.

\section{Resultados e Discussão}

Levando-se em consideração as características clínicas avaliadas e associando-as aos resultados obtidos no exame citológico vaginal, observou-se que das 23 cadelas, 3 encontravam-se em proestro (Figura 3A) e 5 em estro, com predominância de células intermediárias e superficiais, respectivamente. Além disso, 9 cadelas apresentavam-se na fase de diestro e 6 de anestro (Figura 3B), tendo em ambas as fases aumento do número de células parabasais e intermediárias (Tabela 1). Costa (2009) relacionando a condição clínica e citológica vaginal de 85 cadelas, observou que 13 fêmeas apresentavam-se no proestro, 21 no estro, 3 no diestro e 48 no anestro.

Tabela 1 - Número (\%) de fêmeas caninas (Canis lupus familiaris) observadas nas diferentes fases do ciclo estral e que receberam administração de anticoncepcional em Belém, Pará.

\begin{tabular}{|c|c|c|}
\hline Fase'do $\cdot$ Ciclo $\cdot$ Estrala & Número·(\%)·de·Fêmeasa & Uso'de'Anticoncepcionala \\
\hline Proestro $\propto$ & $3 \cdot(13,04) \mathbb{a}$ & 10 \\
\hline Estroo & $5 \cdot(21,73) \propto$ & 30 \\
\hline Diestro & $9 \cdot(39,13) \mathfrak{d}$ & $-\alpha$ \\
\hline Anestro & $6 \cdot(26,08) \mathfrak{d}$ & $-\alpha$ \\
\hline
\end{tabular}




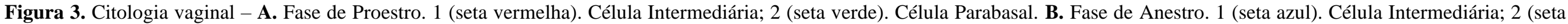
amarela). Célula Parabasal.
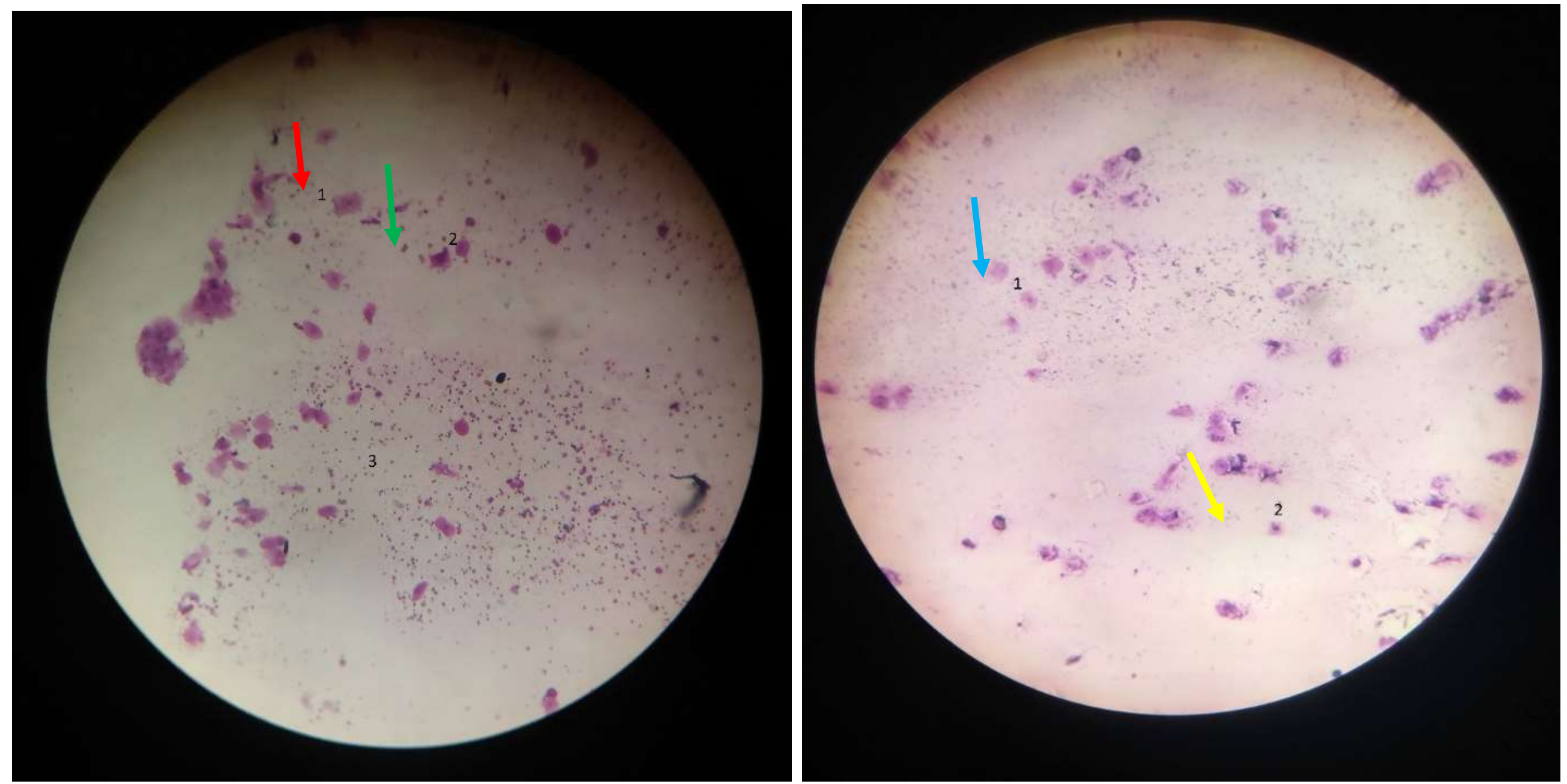

Fonte: Acervo dos autores. 
Sabe-se que as alterações celulares morfológicas no epitélio vaginal ocorrem em decorrência de mudanças hormonais. Alan et al. (2007) e Solano-Gallego; Masserdotti (2016) afirmam que no proestro o aumento da concentração de estrógeno estimula a proliferação do epitélio vaginal e portanto, aumento da sua espessura (de 20 a 30 até 100-150 camadas).

Buen de Argüero (2001), Allison et al. (2008) e Nogueira et al. (2019), descrevem que no estro devido aos estímulos progesterônicos, há predominância de células superficiais poliédricas e com núcleo picnótico, podendo ser detectado também células intermediárias. Já em relação ao diestro e anestro há predomínio de células parabasais e intermediárias arredondadas, com reduzida quantidade ou ausência de bactérias e neutrófilos, respectivamente (Fontbonne; Malandain, 2006; Allison et al., 2008; Payan Carreira; Bessa, 2010; Vieira; Oliveira, 2012). Sendo essa descrição celular morfológica compatível com os resultados obtidos no presente estudo.

Por meio da avaliação do histórico reprodutivo das 23 cadelas, constatou-se que 17,39\% receberam administração de anticoncepcional, sendo detectado 1 fêmea no proestro e 3 no estro (Tabela 1). As fêmeas submetidas ao método contraceptivo apresentaram na avaliação microscópica uma hipoplasia celular, ou seja, redução da população de células epiteliais vaginais quando comparadas aos demais animais. Neste contexto, Christiansen (1988) afirma que o uso de anticoncepcional inibe a síntese e liberação de hormônios reprodutivos como o folículo-estimulante (FSH), o hormônio luteinizante (LH) e a prolactina (PRL), portanto não ocorre o crescimento folicular, a secreção de estrógeno e nem a ovulação, o que resultaria também em pouca ou inexistente alteração na quantidade de células detectadas no exame citológico.

Quanto a idade, observou-se que a maior parte das fêmeas (12/52,17\%) possuíam entre 1 a 5 anos (Tabela 2). Esses resultados estão de acordo com o descrito por Payan Carreira; Bessa (2010) que afirmam que a puberdade das fêmeas ocorre entre 5 a 12 meses de idade quando as mesmas atingem $65 \%$ do peso corporal adulto.

Tabela 2 - Relação entre a idade e a fase do ciclo estral de fêmeas caninas (Canis lupus familiaris) em Belém, Pará.

\begin{tabular}{ccccc}
\hline Fase do Ciclo Estral & $<\mathbf{1}$ ano & $\begin{array}{c}\text { Entre } \\
\mathbf{1} \text { e } \mathbf{5} \text { anos }\end{array}$ & $\begin{array}{c}\text { Entre } \\
\mathbf{6} \text { e 10 anos }\end{array}$ & $>\mathbf{1 1}$ anos \\
\hline Proestro & 1 & 1 & - & 1 \\
Estro & 1 & 4 & - & - \\
Diestro & - & 4 & 3 & - \\
Anestro & 3 & 3 & 3 & 3 \\
Total & 5 & 12 & & - \\
\hline
\end{tabular}

Fonte: Autores.

Todas as fêmeas foram submetidas a avaliação do escore de condição corporal (ECC, variando de 1 a 5), sendo realizado também a relação entre esse índice e a fase do ciclo estral. É interessante ressaltar que 11 (47,82\%) fêmeas apresentaram ECC de 3, sendo considerado ideal (Tabela 3). O ECC é importante devido sua influência sobre as taxas reprodutivas dos animais. Segundo Rhodes et al. (1996), em casos de baixo ECC, ou seja, desnutrição pode haver supressão dos hormônios reprodutivos e manutenção das fêmeas em anestro. Em situações de obsesidade, portanto quando há aumento do ECC, as fêmeas podem apresentar ciclos irregulares e baixa taxa de prenhez (Zoran, 2010). 
Research, Society and Development, v. 10, n. 9, e25310917921, 2021

(CC BY 4.0) | ISSN 2525-3409 | DOI: http://dx.doi.org/10.33448/rsd-v10i9.17921

Tabela 3 - Relação entre o escore de condição coproral (ECC) e a fase do ciclo estral de fêmeas caninas (Canis lupus familiaris) em Belém, Pará.

\begin{tabular}{|c|c|c|c|c|c|}
\hline \multirow[b]{2}{*}{ Fase do Ciclo Estral } & \multicolumn{4}{|c|}{ ECC } & \multirow[b]{2}{*}{5} \\
\hline & 1 & 2 & 3 & 4 & \\
\hline Proestro & - & - & 1 & 2 & 1 \\
\hline Estro & - & 2 & 1 & 1 & - \\
\hline Diestro & - & 1 & 5 & 1 & 2 \\
\hline Anestro & - & - & 4 & 2 & - \\
\hline Total & 0 & 3 & 11 & 6 & 3 \\
\hline
\end{tabular}

Fonte: Autores.

\section{Conclusão}

Foi constatado que a maior parte das cadelas submetidas a avaliação clínica, ginecológica e citológica apresentavamse na fase de diestro, não receberam administração de anticoncepcional, tinham idade superior a 1 ano e ECC de 3, considerado ideal para a reprodução. Diante deste contexto, evidencia-se que a citologia vaginal pode ser empregada na rotina clínica de fêmeas caninas para diagnóstico da fase do ciclo estral, sendo um exame de baixo custo, rápido e simples de ser realizado. Ressalta-se que a associação com a avaliação clínica e hormonal favorece maior precisão nesse diagnóstico.

\section{Referências}

Alan, M. et al. (2007). True vaginal prolapse in a bitch. Animal reproduction science. 100(4), 411- 414

Allison, R. W.; Thrall, M. A.; Olson, P. N. Vaginal Cytology. In: Cowell, R. L.; Tyler, R. D.; Meinkoth, J. H.; Denicola, D. B. (2008). Diagnostic cytology and hematology of the dog and cat. 3.ed. Mosby Elsevier, Missouri. 2008, cap. 25, p. 378-389.

Alves, I.; Mateus, M.; Lopes, L. C. (2002). Monitorização do ciclo éstrico da cadela para inseminação artificial ou cruzamento. In: Congresso de ciências veterinárias. Lisboa: CIISA/Faculdade de Medicina Veterinária, 2002. p. 177-182.

Buen, N. A. (2001). Citología diagnóstica veterinária. El Manual Moderno, Méjico. 137p.

Carvalho, R. L. S.; Pessanha, LDR. (2013). Relação entre famílias, animais de estimação, afetividade e consumo: estudo realizado em bairros do Rio de Janeiro. Sociais e Humanas, 26(3), 622-637.

Chirinéa, V. H. (2008). Inseminação artificial com sêmen congelado em cães. Tese apresentada a Faculdade de Medicina Veterinária e Zootecnia da Universidade Estadual Paulista, UNESP, Campus de Botucatu, para obtenção do título de doutor em Medicina Veterinária, área de Reprodução Animal.

Christiansen, I. B. J. (1988). Reprodução no Cão e Gato. São Paulo: Ed. Manole, 361p.

Concannon, P. W.; Verstegen, J. (2005). Some uniques aspects of canine and feline female reproduction important in veterinary pratice. In: World Small Animal Veterinary Association, 30, 2005, México. Proceedings ... México: WSAVA, p.1-8.

Eynaud, K. et al. (2005). In vivo meiotic resumption, fertilization and early embryonic development in the bitch. Reproduction, 130(2), 193-201.

Feldman, E. C.; Nelson, RW. (2004). Canine and feline endocrinology and reproduction. Ed. W. B. Saunders Company, cap.17, p.785. Pennsylvania: 2004.

Fontbonne, A.; Malandain, E. (2006). Ovarian ultrasonography and follow-up of estrus in the bitch and queen. Waltham Focus, 16(2), 22-29.

Henson, KL. (2003). Sistema reprodutor. In: Raskin, R. E.; Meyer, D. J. Atlas de citologia de cães e gatos. São Paulo: Roca, cap. 11, 233-263p.

IBGE - Instituto Brasileiro De Geografia E Estatística. (2019). Censo Pet, São Paulo.

Klein, B. G. (2014). Cunningham: tratado de fisiologia veterinária. 5.ed. Rio de Janeiro: Elsevier, 416p. 
Research, Society and Development, v. 10, n. 9, e25310917921, 2021

(CC BY 4.0) | ISSN 2525-3409 | DOI: http://dx.doi.org/10.33448/rsd-v10i9.17921

Lúcio, F. M. S. (2018). Apoptose folicular em cadelas e gatas ao longo do ciclo éstrico: apoptose folicular em cadelas e gatas ao longo do ciclo éstrico. $63 \mathrm{f}$ Dissertação (Mestrado) - Curso de Medicina Veterinária, Medicina Veterinária, na área de Clínica Médica e Cirúrgica de Animais de Companhia., Universidade Lusófona de Humanidades e Tecnologias, Lisboa, 2018.

Noakes, D. E.; Parkinson, T. J.; England, GC. (2009). Veterinary reprodution and obstetrics. Saunders.

Nogueira, C. S. et al. (2019). Determinação da fase do ciclo estral através da anamnese e citologia vaginal associada à dosagens hormonais: determination of estrous phase through anamnesis and vaginal cytology associated with hormonal dosages. Brazilian Journal Of Animal And Environmental Research, 2(3), $1037-1045$

Oliveira, A. F. F. (2018). Determinação do período fértil em cadelas por meio de citologia vaginal e dosagem sérica de progesterona. $42 f$. TCC (Graduação) - Curso de Medicina Veterinária, Medicina Veterinária, Centro Universitário de Formiga - Unifor, Formiga.

Oliveira, E. C. S.; Marques, A. P. (2006). Endocrinologia reprodutiva e controle da fertilidade da cadela. Revista Brasileira de Reprodução Animal, 30(2), 1118

Payan-Carreira, R.; Miranda, S.; Nizanski. (2011). Artificial insemination in dogs. 10.5772/20630.

Payan-Carreira, R.; Bessa, A. (2010). Função reprodutiva na cadela. O Cão nº 10 (2010), 2-11.

Rhodes, F. M. et al. (1996). Changes in ovarion function and gonadotropin secretion precending the onset of nutritionally induced anestrus in Bos indicus heifers. Biology Reproduction, 55, 1437-1443.

Serafim, B. K. M. (2009). Cultivo de folículos pré-antrais canídeos em diferentes concentrações de hormônio folículo estimulante. Monografia realizada durante a disciplina Seminários II do programa de pós-graduação em Medicina Veterinária. Área de Concentração em Reprodução Animal da Faculdade de Medicina Veterinária e Zootecnia da Universidade Estadual Paulista (UNESP), campus de Botucatu.

Solano-Gallego, L.; Masserdotti, C. (2016). Canine and feline cytology, a color atlas and interpretation guide. Missouri: Elsevier.

Silva, G. F. (2016). Alterações dermatológicas decorrentes da fase folicular do ciclo estral ou associadas a cistos foliculares ovarianos em cadelas jovens. In: VII Congresso de iniciação científica da FEPI, Itajuba. Pesquisa científica, oportunidade e desafios. Itajuba, p. 1-5.

Vieira, M. et al. (2012). Detecção do ciclo estral por meio de citologia vaginal de cadelas atendidas no hospital veterinário da Univiçosa/Facisa. Anais IV SIMPAC, Revista Científica Univiçosa, 4(1), 143-148.

Zoran, D. (2010). Obesity in dogs and cats: a metabolic and endocrine disorder. Veterinary Clinics of North America: Small Animal Practice, 40(2), 221-239. 